\title{
DISCOVERY OF TeV GAMMA-RAY EMISSION TOWARD SUPERNOVA REMNANT SNR G78.2+2.1
}

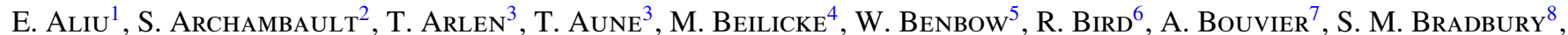

J. H. Buckley ${ }^{4}$, V. Bugaev ${ }^{4}$, K. Byrum ${ }^{9}$, A. CAnnon $^{6}$, A. Cesarini ${ }^{10}$, L. CiupiK ${ }^{11}$, E. Collins-Hughes ${ }^{6}$, M. P. Connolly ${ }^{10}$, W. Cui ${ }^{12}$, R. Dickherber ${ }^{4}$, C. Duke ${ }^{13}$, J. Dumm ${ }^{14}$, V. V. Dwarkadas ${ }^{15}$, M. Errando ${ }^{1}$, A. Falcone ${ }^{16}$, S. Federici ${ }^{17,18}$, Q. Feng ${ }^{12}$, J. P. Finley ${ }^{12}$, G. Finnegan ${ }^{19}$, L. ForTson ${ }^{14}$, A. Furniss ${ }^{7}$, N. Galante ${ }^{5}$, D. Gall ${ }^{20}$, G. H. Gillanders ${ }^{10}$, S. Godambe ${ }^{19}$, E. V. Gotthelf ${ }^{21}$, S. Griffin ${ }^{2}$, J. Grube ${ }^{11}$, G. Gyuk ${ }^{11}$, D. Hanna ${ }^{2}$, J. Holder ${ }^{22}$, H. Huan ${ }^{23}$, G. Hughes ${ }^{17}$, T. B. Humensky ${ }^{24}$, P. KaAReT ${ }^{20}$, N. Karlsson ${ }^{14}$, M. KertZman ${ }^{25}$, Y. Khassen ${ }^{6}$, D. Kieda ${ }^{19}$, H. KrawCZynski ${ }^{4}$, F. Krennrich ${ }^{26}$, M. J. Lang ${ }^{10}$, K. LeE ${ }^{4}$, A. S Madhavan ${ }^{26}$, G. Maier ${ }^{17}$, P. Majumdar ${ }^{3,27}$, S. McArthur ${ }^{23}$, A. McCann ${ }^{28}$, J. Millis $^{29}$, P. Moriarty ${ }^{30}$, R. Mukherjee ${ }^{1}$, T. Nelson ${ }^{14}$, A. O'Faoláin de Bhróithe ${ }^{6}$, R. A. OnG ${ }^{3}$, M. OrR ${ }^{26}$, A. N. Otte ${ }^{31}$, D. Pandel ${ }^{32}$, N. Park ${ }^{23}$, J. S. Perkins ${ }^{33,34}$, M. Pohl ${ }^{17,18}$, A. Popkow ${ }^{3}$, H. Prokoph ${ }^{17}$, J. Quinn ${ }^{6}$, K. Ragan ${ }^{2}$, L. C. Reyes ${ }^{35}$,

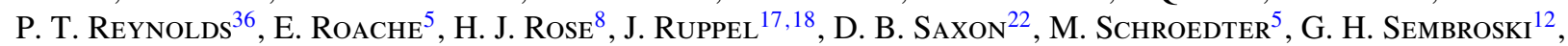
G. D. Şentürk ${ }^{24}$, C. Skole ${ }^{17}$, I. Telezhinsky ${ }^{17,18}$, G. TešIĆ ${ }^{2}$, M. Theiling ${ }^{12}$, S. Thibadeau $^{4}$, K. Tsurusaki $^{20}$, J. TyleR $^{2}$, A. VArlotta ${ }^{12}$, V. V. VAssiliev ${ }^{3}$, S. Vincent ${ }^{17}$, S. P. Wakely ${ }^{23}$, J. E. WARD ${ }^{4}$, T. C. Weekes ${ }^{5}$, A. Weinstein ${ }^{26}$,

T. Weisgarber ${ }^{23}$, R. Welsing ${ }^{17}$, D. A. Williams ${ }^{7}$, AND B. ZitZER ${ }^{9}$

${ }^{1}$ Department of Physics and Astronomy, Barnard College, Columbia University, NY 10027, USA

${ }^{2}$ Physics Department, McGill University, Montreal, QC H3A 2T8, Canada

${ }^{3}$ Department of Physics and Astronomy, University of California, Los Angeles, CA 90095, USA

${ }^{4}$ Department of Physics, Washington University, St. Louis, MO 63130, USA

${ }^{5}$ Fred Lawrence Whipple Observatory, Harvard-Smithsonian Center for Astrophysics, Amado, AZ 85645, USA

${ }^{6}$ School of Physics, University College Dublin, Belfield, Dublin 4, Ireland

${ }^{7}$ Santa Cruz Institute for Particle Physics and Department of Physics, University of California, Santa Cruz, CA 95064, USA

${ }^{8}$ School of Physics and Astronomy, University of Leeds, Leeds, LS2 9JT, UK

${ }^{9}$ Argonne National Laboratory, 9700 S. Cass Avenue, Argonne, IL 60439, USA

${ }^{10}$ School of Physics, National University of Ireland Galway, University Road, Galway, Ireland

${ }^{11}$ Astronomy Department, Adler Planetarium and Astronomy Museum, Chicago, IL 60605, USA

${ }^{12}$ Department of Physics, Purdue University, West Lafayette, IN 47907, USA

${ }^{13}$ Department of Physics, Grinnell College, Grinnell, IA 50112-1690, USA

${ }^{14}$ School of Physics and Astronomy, University of Minnesota, Minneapolis, MN 55455, USA

${ }^{15}$ Department of Astronomy and Astrophysics, University of Chicago, Chicago, IL 60637, USA

${ }^{16}$ Department of Astronomy and Astrophysics, 525 Davey Lab, Pennsylvania State University, University Park, PA 16802, USA

${ }^{17}$ DESY, Platanenallee 6, D-15738 Zeuthen, Germany

${ }^{18}$ Institute of Physics and Astronomy, University of Potsdam, D-14476 Potsdam-Golm, Germany

${ }^{19}$ Department of Physics and Astronomy, University of Utah, Salt Lake City, UT 84112, USA

${ }^{20}$ Department of Physics and Astronomy, University of Iowa, Van Allen Hall, Iowa City, IA 52242, USA

${ }^{21}$ Columbia Astrophysics Laboratory, Columbia University, New York, NY 10027, USA

${ }^{22}$ Department of Physics and Astronomy and the Bartol Research Institute, University of Delaware, Newark, DE 19716, USA

${ }^{23}$ Enrico Fermi Institute, University of Chicago, Chicago, IL 60637, USA

${ }^{24}$ Physics Department, Columbia University, New York, NY 10027, USA

${ }^{25}$ Department of Physics and Astronomy, DePauw University, Greencastle, IN 46135-0037, USA

${ }^{26}$ Department of Physics and Astronomy, Iowa State University, Ames, IA 50011, USA; amandajw @iastate.edu

${ }^{27}$ Saha Institute of Nuclear Physics, 1/AF Bidhannagar, Sector-1 Kolkata-700064, India

${ }^{28}$ Kavli Institute for Cosmological Physics, University of Chicago, Chicago, IL 60637, USA

${ }^{29}$ Department of Physics, Anderson University, 1100 East 5th Street, Anderson, IN 46012, USA

${ }^{30}$ Department of Life and Physical Sciences, Galway-Mayo Institute of Technology, Dublin Road, Galway, Ireland

${ }^{31}$ School of Physics and Center for Relativistic Astrophysics, Georgia Institute of Technology, 837 State Street NW, Atlanta, GA 30332-0430, USA

32 Department of Physics, Grand Valley State University, Allendale, MI 49401, USA

${ }^{33}$ CRESST and Astroparticle Physics Laboratory NASA/GSFC, Greenbelt, MD 20771, USA

${ }^{34}$ University of Maryland, Baltimore County, 1000 Hilltop Circle, Baltimore, MD 21250, USA

${ }^{35}$ Physics Department, California Polytechnic State University, San Luis Obispo, CA 94307, USA

${ }^{36}$ Department of Applied Physics and Instrumentation, Cork Institute of Technology, Bishopstown, Cork, Ireland Received 2013 March 31; accepted 2013 April 18; published 2013 May 29

\begin{abstract}
We report the discovery of an unidentified, extended source of very-high-energy gamma-ray emission, VER J2019+407, within the radio shell of the supernova remnant SNR G78.2+2.1, using $21.4 \mathrm{hr}$ of data taken by the VERITAS gamma-ray observatory in 2009. These data confirm the preliminary indications of gamma-ray emission previously seen in a two-year (2007-2009) blind survey of the Cygnus region by VERITAS. VER J2019+407, which is detected at a post-trials significance of 7.5 standard deviations in the 2009 data, is localized to the northwestern rim

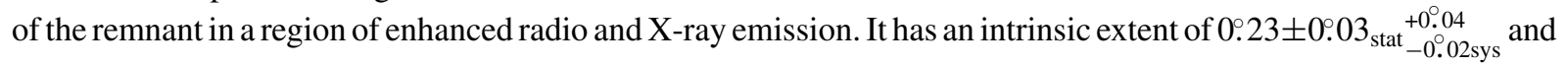
its spectrum is well-characterized by a differential power law $\left(d N / d E=N_{0} \times(E / \mathrm{TeV})^{-\Gamma}\right)$ with a photon index of $\Gamma=2.37 \pm 0.14_{\text {stat }} \pm 0.20_{\text {sys }}$ and a flux normalization of $N_{0}=1.5 \pm 0.2_{\text {stat }} \pm 0.4_{\text {sys }} \times 10^{-12}$ photon $\mathrm{TeV}^{-1} \mathrm{~cm}^{-2} \mathrm{~s}^{-1}$. This yields an integral flux of $5.2 \pm 0.8_{\text {stat }} \pm 1.4_{\text {sys }} \times 10^{-12}$ photon $\mathrm{cm}^{-2} \mathrm{~s}^{-1}$ above $320 \mathrm{GeV}$, corresponding to $3.7 \%$ of the Crab Nebula flux. We consider the relationship of the TeV gamma-ray emission with the GeV gamma-ray emission seen from SNR G78.2+2.1 as well as that seen from a nearby cocoon of freshly accelerated cosmic rays.
\end{abstract}


Multiple scenarios are considered as possible origins for the $\mathrm{TeV}$ gamma-ray emission, including hadronic particle acceleration at the SNR shock.

Key words: acceleration of particles - cosmic rays - gamma rays: general - ISM: supernova remnants

\section{INTRODUCTION}

The stereoscopic imaging atmospheric Cherenkov telescope array VERITAS completed a two-year survey of the Cygnus region of the Galactic Plane in very high energy (VHE) gamma rays (Weinstein et al. 2009). The survey covered the area between Galactic longitudes $67^{\circ}$ and $82^{\circ}$ and Galactic latitudes $-1^{\circ}$ and $4^{\circ}$, a region chosen because it contains both a high density of material and a significant population of potential VHE gamma-ray emitters, including a number of pulsar wind nebulae (PWNe) and supernova remnants (SNRs). As acceleration of either electrons or nuclei above $1 \mathrm{TeV}$ within these stellar remnants can also generate gamma-ray emission, detection of gamma rays from these objects may provide insight into the nature of both the acceleration process within the remnant and the nature of the accelerated population of particles (Blandford \& Eichler 1987; Ellison \& Reynolds 1991; Drury et al. 1994; Ellison et al. 2007). Relativistic electrons can produce gamma rays via both non-thermal bremsstrahlung and inverse-Compton (IC) scattering off nearby optical, IR, or microwave photons. Protons and heavier atomic nuclei generate gamma rays via the decay of neutral pions produced by their interaction with interstellar material. Identifying gamma-ray emission from SNRs that is produced by pion decay could provide insight into the origin of Galactic cosmic rays by providing evidence that SNRs are sites of hadronic cosmic ray acceleration within the Galaxy.

While the initial survey yielded no clear new source detections, the blind search suggested possible VHE gamma-ray emission at several locations, most notably in the vicinity of the SNR G78.2+2.1 ( $\gamma$-Cygni). SNR G78.2+2.1 is a $\sim 1^{\circ}$ diameter $\mathrm{SNR} \sim 1.7 \mathrm{kpc}$ distant, with a shell-like radio and X-ray structure (Higgs et al. 1977; Lozinskaya et al. 2000). It is considered young to middle-aged at $\sim 7000 \mathrm{yr}$ (Higgs et al. 1977; Landecker et al. 1980; Lozinskaya et al. 2000) and in an early phase of adiabatic expansion into a medium of fairly low density (Lozinskaya et al. 2000). Gosachinskij (2001) also identifies a slowly expanding $\mathrm{H}_{\mathrm{I}}$ shell immediately surrounding the radio shell. Lozinskaya et al. (2000) suggest this shell was created by the progenitor stellar wind.

Most of the radio and X-ray emission lies in distinct northern and southern features (Zhang et al. 1997; Uchiyama et al. 2002). The northern region is characterized by enhanced thermal X-ray emission, suggestive of shocked gas (Uchiyama et al. 2002), as well as strong optical emission with sulfur lines (Mavromatakis 2003). However, it evinces little to no CO emission (Ladouceur $\&$ Pineault 2008). The gamma-ray satellite Fermi sees diffuse gamma-ray emission above $10 \mathrm{GeV}$ from the entire remnant (Lande et al. 2012) and has discovered a gamma-ray pulsar, PSR J2021+4026, at the center of the remnant (Abdo et al. 2010a, 2010b). This pulsar, which has a spectral cutoff of $3.0 \mathrm{GeV}$, also has a low luminosity $\left(1.1 \times 10^{35} \mathrm{erg} \mathrm{s}^{-1}\right)$ and a spin-down age $(76.8 \mathrm{kyr})$ much greater than the estimated age of SNR G78.2+2.1. Trepl et al. (2010) argue, however, that PSR J2021+4026 was likely born with something close to its current spin period, in which case its spin-down age is not indicative of its actual age. It therefore remains plausible that
PSR J2021+4026 is the remnant of SNR G78.2+2.1's progenitor star (Trepl et al. 2010). Except where otherwise noted, all GeV and $\mathrm{TeV}$ sources are considered to be at a distance of $1.7 \mathrm{kpc}$, the estimated distance of SNR G78.2+2.1 (Higgs et al. 1977; Lozinskaya et al. 2000).

Subsequent to the completion of the VERITAS Cygnus region survey, follow-up observations were taken of the VHE gamma-ray source candidate near SNR G78.2+2.1. Based on those further observations, we report here the discovery of an extended, unidentified source of VHE gamma rays that lies within an area of enhanced radio emission along the northwestern shell of SNR G78.2+2.1. The observational details are described in Section 2; the analysis and results are presented in Sections 3 and 4, respectively. We discuss the nature of the $\mathrm{TeV}$ gamma-ray emission in Section 5.

\section{OBSERVATIONS}

The VERITAS Observatory consists of an array of four imaging atmospheric Cherenkov telescopes, located at the Fred Lawrence Whipple Observatory in southern Arizona (Holder et al. 2008). Each telescope is equipped with a 3.5 field-ofview (FOV), 499 pixel photomultiplier tube camera. The array is run in a coincident mode, requiring a minimum of two out of four telescopes to trigger in order for an event to be recorded. Data included in this paper were taken with an upgraded array configuration that has improved sensitivity and gamma-ray point-spread function (PSF; Perkins et al. 2009) relative to that used during the Cygnus region survey. This configuration has $\sim 15 \%-25 \%$ energy resolution between $100 \mathrm{GeV}$ and $30 \mathrm{TeV}$ and a $5 \sigma$ (standard deviation above background) point source sensitivity of $1 \%$ of the Crab Nebula flux above $300 \mathrm{GeV}$ in less than $30 \mathrm{hr}$ of observation at zenith angles less than $30^{\circ}$.

The observations under discussion were motivated by, but do not include, data from the VERITAS Cygnus region survey (Weinstein et al. 2009). The initial two-year survey covered the area of the remnant with an effective exposure time of $\sim 30 \mathrm{hr}$ and showed evidence of an extended gamma-ray excess. The approximate location of the survey excess was re-observed with a set of dedicated pointings during 2009 September-November, for a total live-time of $18.6 \mathrm{hr}$. These observations were performed in "wobble" mode, with the center of the VERITAS FOV offset by 0.6 from the target position (R.A. $20^{\mathrm{h}} 19^{\mathrm{m}} 48^{\mathrm{s}}$, decl. $\left.+40^{\circ} 54^{\prime} 00^{\prime \prime}\right)$. The zenith angle was restricted to $10^{\circ}-30^{\circ}$ for these observations. Approximately $10 \%$ of the data were taken at a time when three out of the four VERITAS telescopes were operational; the remaining $90 \%$ was taken with the full array.

\section{ANALYSIS}

Images from these data are calibrated according to the standard procedure described in Cogan et al. (2008) and cleaned using the procedure described in Daniel et al. (2008). After calibration, the primary photon direction is calculated via stereoscopic reconstruction based on the intersection of image primary axes. To ensure that the primary photon direction and energy can be well-reconstructed, an initial event selection is 


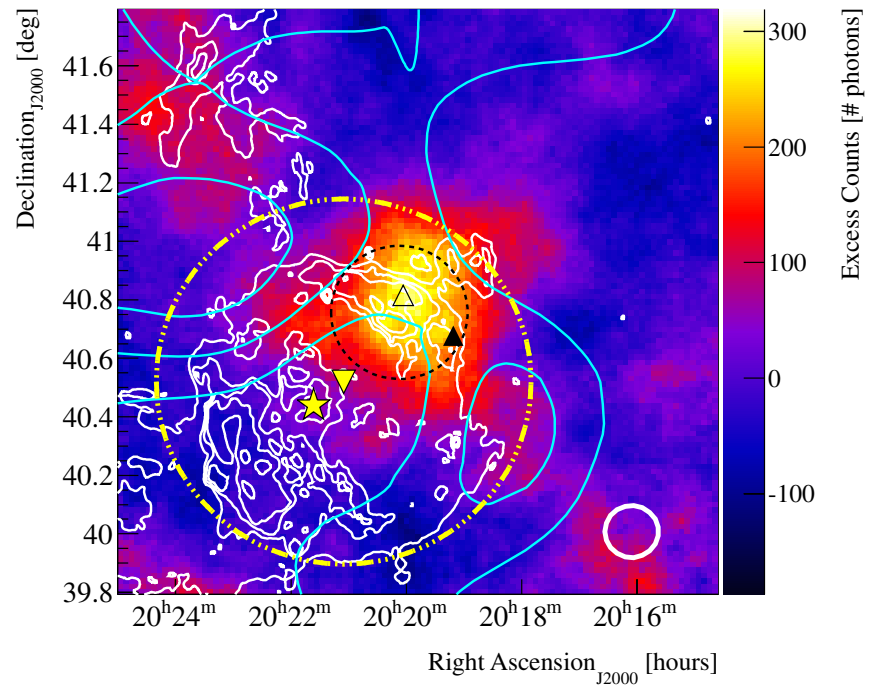

Figure 1. Background-subtracted gamma-ray counts map of SNR G78.2+2.1 showing the VERITAS detection of VER J2019+407 and its fitted extent (black dashed circle). The supernova remnant is delineated by CGPS $1420 \mathrm{MHz}$ continuum radio contours at brightness temperatures of $23.6 \mathrm{~K}, 33.0 \mathrm{~K}, 39.6 \mathrm{~K}$, $50 \mathrm{~K}$, and $100 \mathrm{~K}$ (white; Taylor et al. 2003); the star shows the location of the central gamma-ray pulsar PSR J2021+4026. The inverted triangle and dotdashed circle (yellow) show the fitted centroid and extent of the emission detected by Fermi above $10 \mathrm{GeV}$. The open and filled triangles (black) show the positions of Fermi catalog sources 1FGL J2020.0+4049 and 2FGL J2019.1+4040 which have been subsumed into the extended GeV emission from the entire remnant. The $0.16,0.24$, and 0.32 photons bin $^{-1}$ contours of the Fermi detection of the Cygnus cocoon are shown in cyan. The white circle (bottom right corner) indicates the $68 \%$ containment size of the VERITAS gamma-ray PSF for this analysis.

applied, requiring that events have three images passing the following criteria: more than four pixels per image, an image centroid no more than 1.43 from the camera center, and a total integrated charge per image of at least 70 photoelectrons.

Calibrated images are described in terms of a second-moment parameterization (Hillas 1985). Cosmic-ray background is rejected using selection criteria applied to two composite parameters based on these moments: mean-scaled length (MSL) and mean-scaled width (MSW; Aharonian et al. 1997). We impose the requirements $0.05<\mathrm{MSL}<1.25$ and $0.05<\mathrm{MSW}<1.10$; in addition, we require the angle between the reconstructed gamma-ray arrival direction and the source position to be less than 0.23 . The chosen background-rejection criteria are optimized for moderate-strength ( $\sim 5 \%$ of the Crab nebula flux) extended sources. Together with the image quality requirements they impose an energy threshold for this measurement of $320 \mathrm{GeV}$.

To minimize the number of independent search elements, our search is restricted to a pre-defined circular region with radius 0.25 centered on the target position. In the imaging analysis and source morphology studies the ring background model (Aharonian et al. 2005) is used to estimate the residual cosmic ray background; the reflected-region model (Aharonian et al. 2001) is used when extracting the spectrum. We also excluded from the background estimation circular regions with radius 0.3 around four bright stars in the FOV ( $\gamma$ Cygni, P Cygni, 40 Cygni, and HIP100069) as well as two overlapping circular 0.4 radius regions used to approximate the profile of the excess seen in the VERITAS survey data (Weinstein et al. 2009, 2011). All results reported here have been verified by an independent calibration and analysis chain.

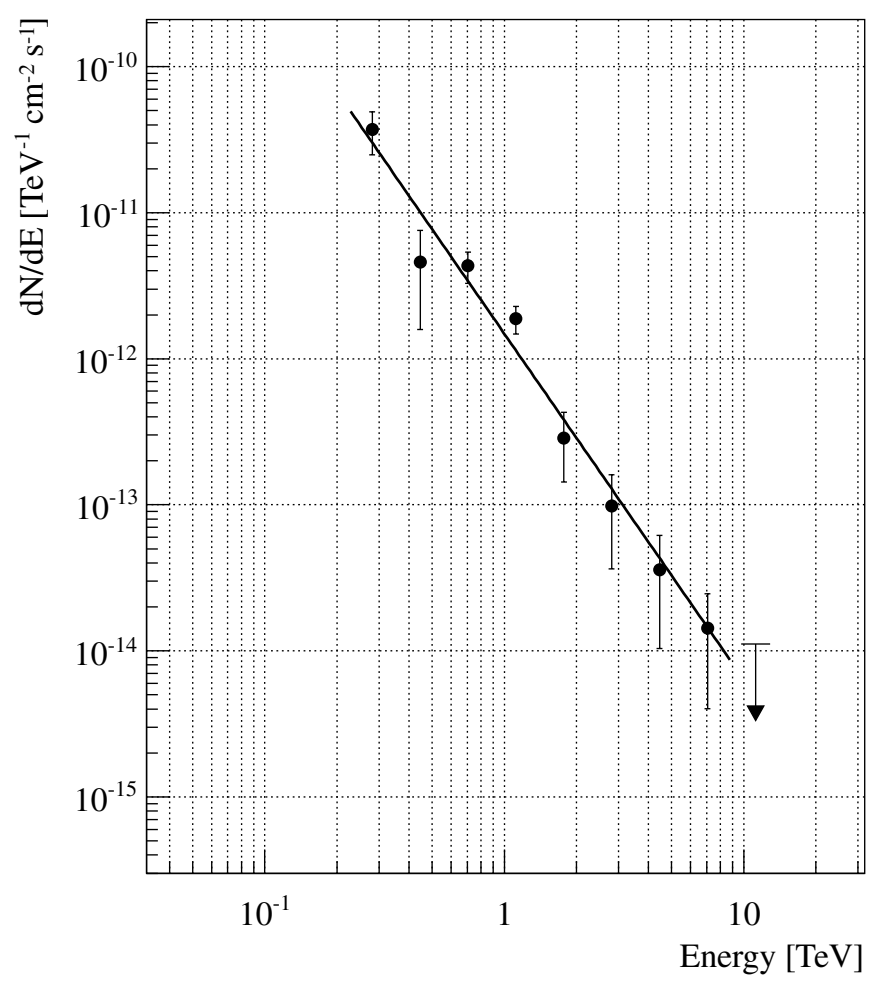

Figure 2. Spectrum of VER J2019+407, derived from four-telescope data only. Points are the VERITAS spectrum, while the arrow indicates the upper limit on emission at $11 \mathrm{TeV}$. The solid line shows a power-law fit with a spectral index of $\Gamma=2.37 \pm 0.14_{\text {stat }} \pm 0.20_{\text {sys }}$ and a flux normalization of $N_{0}=1.5 \pm 0.2_{\text {stat }} \pm 0.4_{\text {sys }} \times 10^{-12}$ photon $\mathrm{TeV}^{-1} \mathrm{~cm}^{-2} \mathrm{~s}^{-1}$.

\section{RESULTS}

Figure 1 displays the background-subtracted, acceptancecorrected TeV image of the region of SNR G78.2+2.1. A clear signal with $319 \pm 39$ net counts is detected at the location of the northern rim of the remnant. This signal is significant at the $7.5 \sigma$ level after accounting for all test points in the pre-defined 0.25 search region. Figure 1 also shows the locations of the gammaray pulsar PSR J2021+4026 (1FGL J2021.5+4026), 0.5 from VER J2019+407 at the center of the SNR, and the centroid of the emission from the remnant seen by Fermi above $10 \mathrm{GeV}$.

The morphology of VER J2019+407 is derived from a binned extended maximum-likelihood fit to the counts map before background subtraction. The cosmic ray component is modeled as an exposure-modulated flat background and the source by a symmetric two-dimensional Gaussian convolved with the VERITAS PSF (68\% containment radius of 0.09 , derived from an identically processed observation of the Crab Nebula). We find a fitted extension of $0.23 \pm 0.03_{\text {stat }_{-0.02 \text { sys }}+0^{\circ} .04}^{\circ}$. The fitted centroid coordinates are R.A. $20^{\mathrm{h}} 20^{\mathrm{m}} 04^{\mathrm{s}} .8$, decl. $+40^{\circ} 45^{\prime} 36^{\prime \prime}$ (J2000); however, we maintain the identifier VER J2019+407 for the source, which was originally assigned on the basis of a preliminary centroid estimation. The statistical uncertainty in this location is 0.03 , with a combined systematic uncertainty in the position, due to the telescope pointing error and systematic errors of the fit itself, of 0.018 .

Figure 2 shows the spectrum derived from the reconstructed gamma-ray events within 0.24 from the center of the search region; runs where only three of four telescopes were operational have been excluded from this sample. The threshold for the spectral analysis is $320 \mathrm{GeV}$ and the energy resolution is $15 \%$ at 


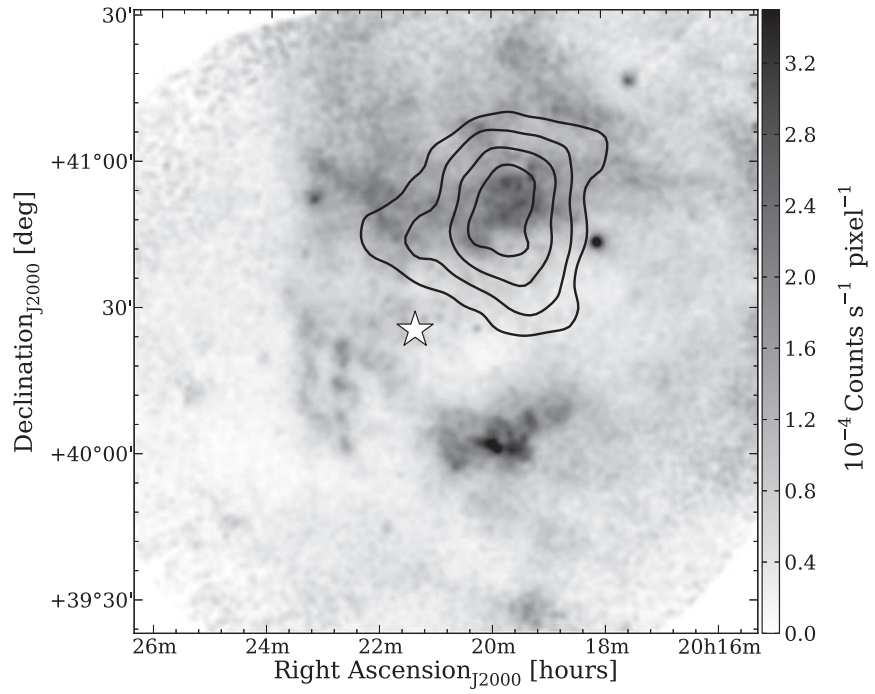

Figure 3. ROSAT PSPC X-ray view of SNR G78.2+2.1 between 1 and $2 \mathrm{keV}$. The VER J2019+407 smoothed photon excess contours (100, 150, 210, and 260 photons) are superimposed. The image is composed of a mosaic of six exposure- and vignette-corrected overlapping observations, smoothed using a $5 \times 5$ pixel boxcar filter. The lower energy bound was selected to reject the background flux from the Galactic Plane. The location of the gamma-ray pulsar PSR J2021+4026 is marked with a white star.

$1 \mathrm{TeV}$. The photon spectrum is fit well $\left(\chi^{2} /\right.$ dof $\left.=8.76 / 6\right)$ by a differential power law in energy, $d N / d E=N_{0} \times$ $(E / \mathrm{TeV})^{-\Gamma}$, between $320 \mathrm{GeV}$ and $10 \mathrm{TeV}$, with a photon index of $2.37 \pm 0.14_{\text {stat }} \pm 0.20_{\text {sys }}$ and a flux normalization at $1 \mathrm{TeV}$ of $N_{0}=1.5 \pm 0.2_{\text {stat }} \pm 0.4_{\text {sys }} \times 10^{-12}$ photon $\mathrm{TeV}^{-1} \mathrm{~cm}^{-2} \mathrm{~s}^{-1}$. The integral flux above $320 \mathrm{GeV}\left(5.2 \pm 0.8_{\text {stat }} \pm 1.4_{\text {sys }} \times\right.$ $10^{-12}$ photon $\mathrm{cm}^{-2} \mathrm{~s}^{-1}$ ) corresponds to $3.7 \%$ of the Crab Nebula flux above that energy. No other significant $\mathrm{TeV}$ source is found in the maps.

\subsection{X-Ray Observations}

Figure 3 presents an exposure-corrected ROSAT PSPC 1-2 keV image of SNR G78.2+2.1 generated from archival data (see Brazier et al. 1996 for observational details). The $\mathrm{TeV}$ gamma-ray emission overlaps a region of enhanced $\mathrm{X}$-ray emission coincident with the bright radio arcs associated with the northern rim of the SNR shell. As the highly absorbing column to the remnant screens out most source photons below $\sim 1 \mathrm{keV}$, we excluded all photons below this energy to suppress background. Several bright X-ray sources are associated with known stars and have been identified in previous work, but none of these overlap the VHE gamma-ray-emitting region (Becker et al. 2004; Weisskopf et al. 2006).

To better study the enhanced X-ray emission overlapping the $\mathrm{TeV}$ contours, we also re-analyzed the data from ASCA Sequence \#25010000 previously studied by Uchiyama et al. (2002). Figure 4 shows the $0.7-3.0 \mathrm{keV}$ exposure-corrected $\mathrm{X}$-ray map, generated by co-adding data from the two gas imaging spectrometers. The image is consistent with that seen by ROSAT. We extracted source and background spectra from the two $12^{\prime} \times 24^{\prime}$ elliptical regions displayed in Figure 4 and generated count-weighted response files appropriate for diffuse emission. The source region, centered on coordinates R.A. $20^{\mathrm{h}} 20^{\mathrm{m}} 17^{\mathrm{s}}$, decl. $+40^{\circ} 45^{\prime} 41^{\prime \prime}(\mathrm{J} 2000)$ and oriented with position angle $60^{\circ}$, contains the bulk of the X-ray emission located within the VERITAS contours. The background ellipse, centered at R.A. $20^{\mathrm{h}} 19^{\mathrm{m}} 38^{\mathrm{s}}$, decl. $+40^{\circ} 27^{\prime} 02^{\prime \prime}(\mathrm{J} 2000)$ with position

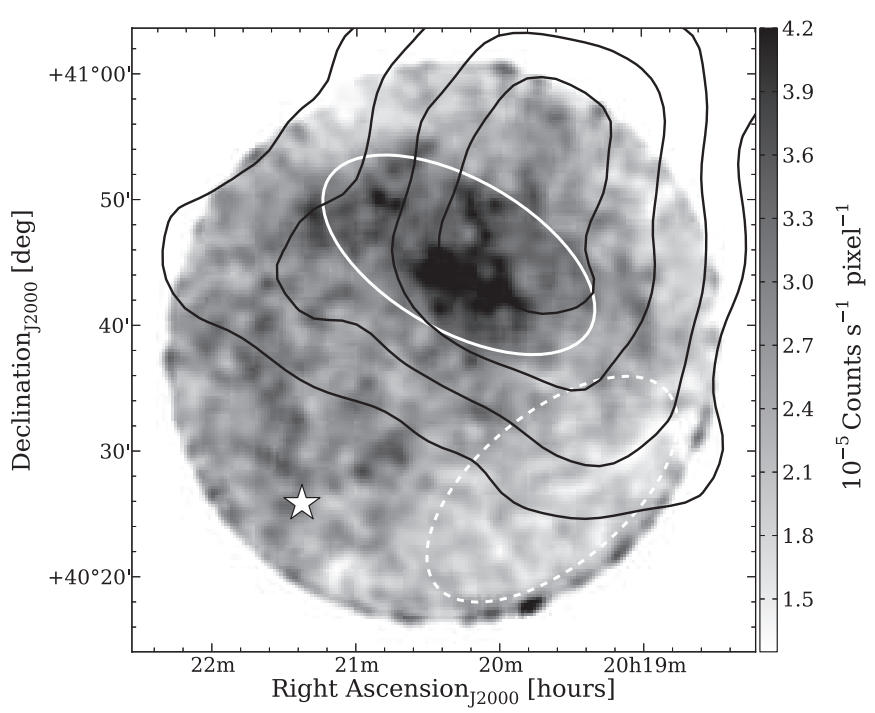

Figure 4. ASCA X-ray view of G78.2+2.1 between 1 and $3 \mathrm{keV}$, overlaid with the VER J2019+407 smoothed photon excess contours $(100,150,210$, and 260 photons). The region used to extract a spectrum and the corresponding background region are indicated by white solid and dashed ellipses, respectively. A white star marks the position of PSR J2021+4026.

angle $130^{\circ}$, is placed where the X-ray emission is evidently at background level in the ROSAT image. The source spectrum was grouped with a minimum of 20 counts per channel and fitted in the $0.7-3.0 \mathrm{keV}$ range using the XSPEC software package. The spectra are evidently soft with few net counts above $\sim 3 \mathrm{keV}$ after background subtraction. A significant line feature is found at $1.9 \mathrm{keV}$; we do not, however, find any evidence for the strong Ne line feature at $\simeq 0.9 \mathrm{keV}$ reported by Uchiyama et al. (2002).

We modeled the X-ray spectrum using an absorbed Raymond-Smith thermal plasma model (see Figure 5). This provides an adequate fit with $\chi^{2} /$ dof $=40.8 / 44$. The bestfitted temperature is $k T=0.57 \pm 0.14 \mathrm{keV}$. Si is overabundant by a factor of $2.0_{-1.2}^{+1.8}$ relative to solar. The column density is $N_{\mathrm{H}}=(3.7 \pm 2.0) \times 10^{21} \mathrm{~cm}^{-2}$ and the normalization is $N=1.8 \times 10^{-3} \mathrm{~cm}^{-5}$. The absorption-corrected flux for this model is found to be $6.0 \times 10^{-12} \mathrm{erg} \mathrm{cm}^{-2} \mathrm{~s}^{-1}$ in the $0.5-8.0 \mathrm{keV}$ band. We also considered that the plasma behind a shock expanding in a low-density medium might not have had time to reach ionization equilibrium given the assumed SNR lifetime, in which case the spectrum would be better modeled by a non-equilibrium ionization (NEI) model. We attempted to fit the ASCA spectrum with an absorbed NEI model, the "vgnei" model of XSPEC, but were unable to obtain good constraints on the fitted parameters.

We also attempted to constrain the flux of any possible powerlaw component in the spectrum by adding a power-law with photon index fixed to 2.0 and then varying the power-law normalization until the $\chi^{2}$ increased by 2.7 (90\% confidence level) relative to the best fitted model with no power-law component. We place an upper limit on the flux of a powerlaw component of $1.9 \times 10^{-12} \mathrm{erg} \mathrm{cm}^{-2} \mathrm{~s}^{-1}$ in the $0.5-8.0 \mathrm{keV}$ band.

We note that our result differs significantly from that given in Uchiyama et al. (2002). While we have chosen a source region similar to their "R1" source region, we find that our fits to the ASCA spectrum require neither an additional power-law component nor a large Ne IX line feature. The discrepancy seems to be caused by the choice of background region. We selected a background region that was as close as possible to the source, in 


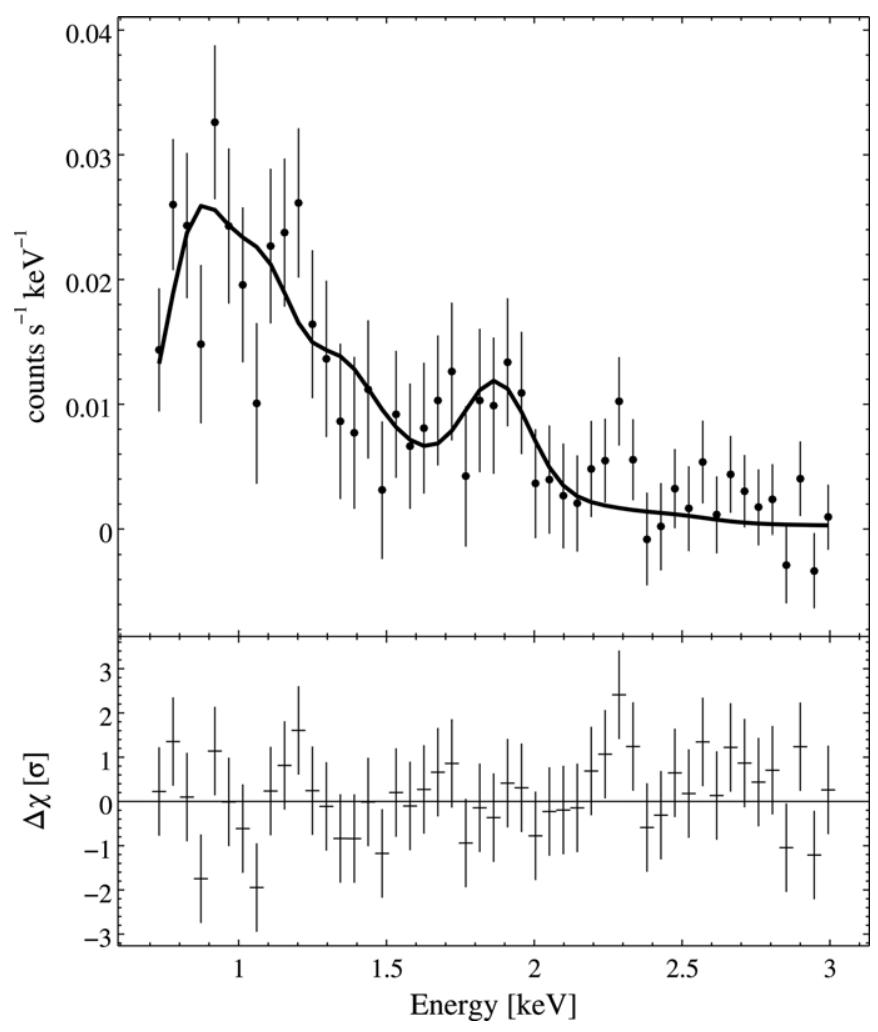

Figure 5. Top panel: ASCA X-ray spectrum of the region of enhanced X-ray emission coincident with VER J2019+407, as shown in Figure 4. The solid line shows the fit of a Raymond-Smith thermal plasma model with parameters as given in the text. We identify the line at $1.9 \mathrm{keV}$ as due to Si. Bottom panel $-\Delta \chi$ residuals (residual divided by the statistical error) for the best-fit model.

order to account for the large variance in the Galactic emission in this part of the plane. Uchiyama et al. (2002) considered four nearby ASCA pointings and chose the one with the least source contamination, $\sim 3.5$ from the center of the "R1" region. A choice of background region similar to Uchiyama's allows us to reproduce the previously reported nonthermal component and spectral features.

\section{DISCUSSION}

We have discovered a spatially extended source of VHE gamma-ray emission, VER J2019+407, located on the northwest rim of the shell-type SNR G78.2+2.1. The TeV gammaray emission is coincident with the leading edge of a prominent arc-shaped radio continuum feature along the SNR rim (Figure 1) and with a region of enhanced X-ray emission that we identify as thermal in origin (Figures 3-5). VER J2019+407 lies near the inner edge of a slowly expanding H I shell immediately surrounding the radio shell identified in $21 \mathrm{~cm}$ line observations (Gosachinskij 2001); it also lies near a region of bright $\left[\mathrm{S}_{\mathrm{II}}\right]$ optical line emission within the SNR that is identified as shock-heated gas based on the $[\mathrm{S} \mathrm{II}] / \mathrm{H} \alpha$ line ratio (Mavromatakis 2003). There are also two GeV sources associated with SNR G78.2+2.1. One is a pulsar, 1FGL J2021.5+4026, located at the center of the remnant (Abdo et al. 2010a, 2010b). The other is an extended source candidate reported above $10 \mathrm{GeV}$ by Fermi (Lande et al. 2012). A point source colocated with VER J2019+407 was previously reported in the first and second Fermi catalogs, but Lande et al. (2012) conclude that it was an artifact of failing to model the extended source above $10 \mathrm{GeV}$ rather than a separate source. Between
G78.2+2.1 and the Cyg OB2 association (roughly 2.4 away) lies a 50 parsec-wide area of $1-100 \mathrm{GeV}$ gamma-ray emission, detected by Fermi, that has been interpreted as a cocoon of freshly accelerated cosmic rays (Ackermann et al. 2011).

The TeV gamma-ray emission and the features observed in the $\mathrm{X}$-ray, optical, and radio continuum can be most simply related to the presence of shocks at the interaction of the supernova ejecta and the surrounding medium. SNR G78.2+2.1 has a relatively low interior density and, as previously noted, appears to be surrounded by a dense H I shell (Gosachinskij 2001). Lozinskaya et al. (2000) suggest that this H I shell was created by the progenitor stellar wind as it swept up the ambient medium. The interaction of the supernova shock with the H I shell might drive a shock into the dense shell and a reflected shock back into the cavity. Emission arising from these shocks would then account for the observed [S II] lines, enhanced thermal X-rays, and strong radio continuum emission near VER J2019+407.

Shocks can produce $\mathrm{TeV}$ gamma-ray emission via either IC scattering of accelerated electrons or hadronic interactions of accelerated nuclei. High-energy electrons capable of producing $\mathrm{TeV}$ photons via IC scattering should also produce X-ray synchrotron radiation detectable as a non-thermal power-law in the X-ray spectrum. While our analysis of the ASCA X-ray spectrum shows no evidence for a non-thermal component, the upper limit on this component is not yet strong enough to rule out IC scattering as the source of the $\mathrm{TeV}$, if not the $\mathrm{GeV}$, gammaray emission. Deeper X-ray observations of VER J2019+407 should provide better constraints.

The flux of $\mathrm{TeV}$ photons from hadronic processes depends on the energy available for shock acceleration and the density of the target material. One of the prerequisites of this scenario is that the necessary density of target material, as inferred from the gamma-ray flux, be consistent with that estimated from measurements at other wavelengths. Equation (9) in Drury et al. (1994) gives (on the assumption that the spectrum of the charged particles is a power law with index of -2) the expected gammaray flux above a given threshold energy as a function of the threshold energy $E$, the fraction $\theta$ of the SN kinetic energy $E_{\mathrm{SN}}$ converted to cosmic rays, the distance $d$ of the SNR, and the density $n$ of the target material,

$$
\begin{aligned}
F(>E) \approx & 910^{-11} \theta\left(\frac{E}{1 \mathrm{TeV}}\right)^{-1.1}\left(\frac{E_{\mathrm{SN}}}{10^{51} \mathrm{erg}}\right) \\
& \times\left(\frac{d}{1 \mathrm{kpc}}\right)^{-2}\left(\frac{n}{1 \mathrm{~cm}^{-3}}\right) \mathrm{cm}^{-2} \mathrm{~s}^{-1} .
\end{aligned}
$$

For $E=320 \mathrm{GeV}, d=1.7 \mathrm{kpc}, \theta \sim 0.1$ as expected for a remnant in the early Sedov phase, and assuming $E_{\mathrm{SN}}=10^{51}$ erg, which is consistent with estimates derived from optical data (Mavromatakis 2003), we find that a density of $0.5 \mathrm{~cm}^{-3}$ is required to produce the observed TeV flux. However, this equation is for the total flux integrated over the whole spherical shell of a SNR. The gamma-ray emission from VER J2019+407 is confined to a relatively small portion of the SNR shell. Thus, it is likely that only a fraction of the SN blast wave participates in the shock acceleration producing the $\mathrm{TeV}$ gamma-ray emission. Assuming an isotropic explosion, we account for this by scaling $E_{\mathrm{SN}}$ by the ratio of the surface area covered by VER J2019+407 to the total surface area of the remnant. Using the fitted $95 \%$ $(68 \%)$ containment radius of the $\mathrm{TeV}$ gamma-ray emission gives a correction factor of $0.5(0.14)$. Once uncertainties in the source extension, integral flux, and SNR distance are also taken into 
account, the average density required to produce the observed $\mathrm{TeV}$ flux above $320 \mathrm{GeV}$ increases to $1.0-5.5 \mathrm{~cm}^{-3}$.

We find these densities consistent with our knowledge of the region from other wavelengths. Given that no significant $\mathrm{CO}$ emission is detected in this area of the remnant, any target material must be atomic rather than molecular, with the necessary high density perhaps arising in the swept-up H i shell. Gosachinskij (2001) estimated a gas density of $2.5 \mathrm{~cm}^{-3}$ in the $\mathrm{H}$ I shell surrounding the remnant, consistent with the range of densities derived from the $\mathrm{TeV}$ data. Optical data also shed some light on densities in the vicinity of VER J2019+407. Mavromatakis gives the ratio of [S II] $\lambda 6716$ to [S II] $\lambda 6731$ line fluxes at multiple locations within G78.2+2.1, one of which is close to (although not precisely coincident with) VER $\mathrm{J} 2019+407$. All of these ratios are close to one, and, within a plausible range of temperatures, imply post-shock densities on the order of a few $100 \mathrm{~cm}^{-3}$. Mavromatakis infers these densities to be arising behind a radiative shock and estimates a primary shock velocity of $\sim 750 \mathrm{~km} \mathrm{~s}^{-1}$, consistent with that inferred from an X-ray temperature of $0.57 \mathrm{keV}$. He deduces pre-shock cloud densities on the order of $\sim 20 \mathrm{~cm}^{-3}$, which are not unreasonable given the average densities inferred here from the gamma-ray flux. The shock velocities inferred from the optical and X-ray data, however, are too low for the forward shock to accelerate particles to $\mathrm{TeV}$ energies at the present time. Any hadronic TeV gamma-ray emission would have to arise from particles accelerated during an earlier epoch that are only now interacting with the shell.

The limited angular extent of the TeV gamma-ray emission also raises questions. If we view the $\mathrm{SN}$ blastwave as expanding in the low-density bubble blown by the progenitor stellar wind until it reaches the much higher-density cavity wall, why should the interaction favor one portion of the cavity wall over the others? Asymmetries in the shock propagation, variations in the density of the cavity wall, or the former presence of a cloud (now evaporated by the shock) within the low density region could provide an explanation, as could the strength and orientation of the ambient magnetic field relative to the local shock velocity (Ellison et al. 2007). Given the more symmetric and diffuse $\mathrm{GeV}$ gamma-ray emission seen from the remnant, asymmetric diffusion of accelerated particles out of the remnant might also play a role, as the highest-energy particles would be the first to escape. However, at this stage there is no clear evidence for or against any of these possibilities.

In these scenarios, SNR G78.2+2.1 could be responsible for some or all of the freshly accelerated cosmic rays within the cocoon detected by Fermi. However, the results shown here cannot be used to draw strong conclusions about the cocoon's relationship to SNR G78.2+2.1 or to set a meaningful upper limit on cocoon emission above $300 \mathrm{GeV}$. The cocoon contours from Ackermann et al. (2011) were derived from an analysis where 1FGL J2020.0+4049, which is no longer considered an independent source, was included as part of the background model. Since this would artificially reduce the intensity of the GeV gamma-ray emission seen near VER J2019+407, the Ackermann et al. (2011) contours provide a spatial reference only and should not be used to judge the relationship of the cocoon to VER J2019+407. Moreover, gamma-ray emission on the scale of the cocoon (roughly $4 \mathrm{deg}^{2}$ ) cannot be detected with VERITAS using the ring-background estimation method, which will cause the source to self-subtract. Analysis techniques better adapted to highly extended sources, combined with further data taken in alternative observation modes, will be required to confirm the presence or absence of cocoon emission and make a definitive statement about SNR G78.2+2.1's role in feeding the cocoon.

VER J2019+407 bears some similarity to other well known TeV sources. H.E.S.S. observations of the shell-type SNR RCW 86 have revealed the presence of VHE gamma rays suggestive of a shell-type morphology (Aharonian et al. 2009). Berezhko et al. (2009) have explained the $\mathrm{TeV}$ gamma-ray emission from RX J0852.0-4622 as arising from hadronic emission in a wind-bubble scenario, similar in many aspects to the scenario proposed here.

Other explanations of VER J2019+407 are also possible. Nearly half of Galactic TeV sources are identified with PWNe. TeV PWNe are typically extended sources energized by electrons accelerated in the pulsar wind. Because the lifetimes of the electrons can be long, the electrons can diffuse over large distances from the pulsar and the centroid of the TeV gamma-ray emission is often offset from the pulsar (Hessels et al. 2008). On the one hand, the luminosity of VER J2019+407 in the $1-10 \mathrm{TeV}$ band $-2.5 \times 10^{33} \mathrm{erg} \mathrm{s}^{-1}$ assuming a distance of $1.7 \mathrm{kpc}$-is $2 \%$ of the spin-down power of PSR J2021+4026, a value within the range seen for PWNe. On the other hand, Hessels et al. (2008) examined the offsets between $\mathrm{TeV}$ gamma-ray emission and pulsar location for $21 \mathrm{TeV}$ sources, and the pulsar, while offset from the centroid of the $\mathrm{TeV}$ gamma-ray emission, generally lies well within the angular extent of the TeV source. In the single case for which the angular offset is significantly larger than the angular size of the TeV source-PSR J1702-4128/HESS J1702-420 - inspection of the TeV image shows $\mathrm{TeV}$ emission at the pulsar location (Aharonian et al. 2008). By contrast, the offset between PSR J2021+4026 and VER J2019+407 is several times larger than the angular size of VER J2019+407, and we detect no $\mathrm{TeV}$ gamma-ray emission either at the location of PSR J2021+4026 or in the intervening region between PSR $\mathrm{J} 2021+4026$ and the TeV source.

Finally, it is possible that VER J2019+407 is the PWN of an unknown pulsar in the line-of-sight toward SNR G78.2+2.1. This scenario could explain the $\mathrm{GeV}$ and radio continuum emission, but would ascribe the location of VER J2019+407 near the $[\mathrm{S} \mathrm{II}]$ line emission and the enhanced thermal X-ray emission within SNR G78.2+2.1 to chance superposition. Such a scenario cannot be excluded at the current time, but could be tested by a sensitive search for the PWN in the X-ray band and also by radio or X-ray searches for the putative pulsar.

In summary, we have detected gamma-ray emission from a region of enhanced radio, optical line, and X-ray emission in the northwestern shell of SNR G78.2+2.1. The extended $\mathrm{TeV}$ source overlaps with $\mathrm{GeV}$ gamma-ray emission from the remnant but is notably offset from the gamma-ray pulsar PSR J2021+4026 which lies outside the $99 \%$ confidence contour of VER J2019+407. It seems most probable that VER J2019+407 arises from particles (either hadronic or leptonic) accelerated within the SNR shock, although we cannot yet rule out the possibility of a line-of-sight coincidence between the remnant and an un-associated PWN. Deeper high energy observations are needed to better constrain possible emission models.

This research is supported by grants from the U.S. Department of Energy Office of Science, the U.S. National Science Foundation and the Smithsonian Institution, by NSERC in Canada, by the Science Foundation Ireland (SFI 10/RFP/AST2748) and by the Science and Technology Facilities Council in the UK. We acknowledge the excellent work of the technical support 
staff at the Fred Lawrence Whipple Observatory and at the collaborating institutions in the construction and operation of the instrument. Dr. Weinstein and Dr. Dwarkadas' research was also supported in part by NASA grant NNX11A086G.

\section{REFERENCES}

Abdo, A. A., Ackermann, M., Ajello, M., et al. 2010a, ApJS, 187, 460 Abdo, A. A., Ackermann, M., Ajello, M., et al. 2010b, ApJS, 188, 405 Ackermann, M., Ajello, M., Allafort, A., et al. 2011, Sci, 334, 1103 Aharonian, F. A., Akhperjanian, A. G., Aye, K.-M., et al. 2005, A\&A, 430, 865 Aharonian, F. A., Akhperjanian, A. G., Barres de Almeida, U., et al. 2008, A\&A, 477,353

Aharonian, F. A., Akhperjanian, A. G., Barres de Almeida, U., et al. 2009, ApJ, 692, 1500

Aharonian, F. A., Akhperjanian, A., Barrio, J., et al. 2001, A\&A, 370, 112

Aharonian, F. A., Hofmann, W., Konopelko, A. K., \& Völk, H. J. 1997, APh, 6,343

Becker, W., Weisskopf, M., Arzoumanian, Z., et al. 2004, ApJ, 615, 897

Berezhko, E. G., Puhlhofer, G., \& Völk, H. J. 2009, A\&A, 505, 641

Blandford, R., \& Eichler, D. 1987, PhR, 154, 1

Brazier, K. T. S., Kanbach, G., Carramiñana, A., Guichard, J., \& Merck, M. 1996, MNRAS, 281, 1033

Cogan, P., Acciari, V. A., Amini, R., et al. 2008, in Proc. 30th Int. Cosmic Ray Conf., Vol. 3, ed. R. Caballero, J. C. D’Olivo, G. Median-Tanco, L. Nellen, F. A. Sánchez, \& J. F. Valdés-Galicia (Mexico City, Mexico: Universidad Nacional Autónoma de México), 1385

Daniel, M. K., Acciari, V. A., Amini, R., et al. 2008, in Proc. 30th Int. Cosmic Ray Conf., Vol. 3, ed. R. Caballero, J. C. D’Olivo, G. Median-Tanco, L. Nellen, F. A. Sánchez, \& J. F. Valdés-Galicia (Mexico City, Mexico: Universidad Nacional Autónoma de México), 1325
Drury, L. O'C., Aharonian, F. A., \& Völk, H. J. 1994, A\&A, 287, 959

Ellison, D. C., Patnaude, D. J., Slane, P., Blasi, P., \& Gabici, S. 2007, ApJ, 661, 879

Ellison, D. C., \& Reynolds, S. P. 1991, ApJ, 382, 242

Gosachinskij, I. V. 2001, AstL, 27, 233

Hessels, J. W. T., Nice, D. J., Gaensler, B. M., et al. 2008, ApJL, 682, L41

Higgs, L. A., Landecker, T. L., \& Roger, R. S. 1977, AJ, 82, 718

Hillas, A. M. 1985, in Proc. 19th Int. Cosmic Ray Conf., Vol. 3, ed. F. C. Jones, 445

Holder, J., Acciari, V. A., Aliu, E., et al. 2008, in AIP Conf. Proc. 1085, Proc. 4th Int. Meeting on High Energy Gamma-Ray Astronomy, ed. F. A. Aharonian, W. Hofmann, \& F. Rieger (Melville, NY: AIP), 657

Ladouceur, Y., \& Pineault, S. 2008, A\&A, 490, 197

Lande, J., Ackermann, M., Allafort, A., et al. 2012, ApJ, 756, 5

Landecker, T. L., Roger, R. S., \& Higgs, L. A. 1980, A\&AS, 39, 133

Lozinskaya, T. A., Pravdikova, V. V., \& Finoguenov, A. V. 2000, AstL, 26, 77

Mavromatakis, F. 2003, A\&A, 408, 237

Perkins, J. S., Maier, G., Aliu, E., et al. 2009, in 2009 Fermi Symposium, Washington, DC, ed. W. N. Johnson \& D. J. Thompson, eConf C0911022, arXiv:0912.3841

Taylor, A. R., Gibson, S. J., Peracaula, M., et al. 2003, AJ, 125, 3145

Trepl, L., Hui, C. Y., Cheng, K. S., et al. 2010, MNRAS, 405, 1339

Uchiyama, Y., Takahashi, T., Aharonian, F. A., \& Mattox, J. R. 2002, ApJ, 571,866

Weinstein, A., Aliu, E., Arlen, T., et al. 2009, in Fermi Symposium, Washington, DC, ed. W. N. Johnson \& D. J. Thompson, eConf C0911022, arXiv:0912.4492

Weinstein, A., Aliu, E., Arlen, T., et al. 2011, in Proc. 32nd Int. Cosmic Ray Conf., Vol. 7, 162

Weisskopf, M. C., Swartz, D. A., Carramiñana, A., et al. 2006, ApJ, 652,387

Zhang, X., Zheng, Y., Landecker, T. L., \& Higgs, L. A. 1997, A\&A, 324, 641 